\title{
Interactions between Prepregnancy Overweight and Passive Smoking for Macrosomia and Large for Gestational Age in Chinese Pregnant Women
}

\author{
Dingyu Cui ${ }^{a}$ Wen Yang ${ }^{a}$ Ping Shao $^{b}$ Jing Li $^{a, c, d}$ Peng Wang ${ }^{b}$ \\ Junhong Leng ${ }^{e}$ Shuo Wang ${ }^{b}$ Enqing Liu ${ }^{e}$ Juliana C.N. Chan ${ }^{f}$ Zhijie Yu $^{g}$ \\ Gang Hu ${ }^{\text {h }}$ Xilin Yang ${ }^{\mathrm{a}, \mathrm{c}} \mathrm{d}$
}

\begin{abstract}
aDepartment of Epidemiology and Biostatistics, School of Public Health, Tianjin Medical University, Tianjin, China; bProject Office, Tianjin Women and Children's Health Center, Tianjin, China; 'Tianjin Key Laboratory of Environment, Nutrition and Public Health, Tianjin, China; 'Tianjin Center for International Collaborative Research on Environment,

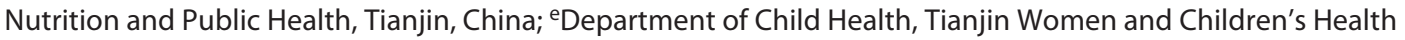
Center, Tianjin, China; 'Department of Medicine and Therapeutics, Hong Kong Institute of Diabetes and Obesity and The Chinese University of Hong Kong-Prince of Wales Hospital-International Diabetes Federation Centre of Education, The Chinese University of Hong Kong, Hong Kong SAR, China; 9Population Cancer Research Program,

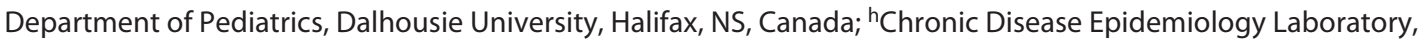
Pennington Biomedical Research Center, Baton Rouge, LA, USA
\end{abstract}

\section{Keywords}

Chinese - Overweight · Passive smoking · Birth weight · Macrosomia

\begin{abstract}
Introduction: Previous analysis showed that passive smoking and overweight were associated with an increased risk of gestational diabetes mellitus (GDM) in a synergistic manner, while GDM increased the risk of macrosomia/large for gestational age (LGA). This study aimed to examine any interactive effects between passive smoking and overweight/ obesity on risk of macrosomia/LGA. Methods: From 2010 to $2012,22,302$ pregnant women registered for pregnancy at a primary hospital in Tianjin, China. Data were collected longitudinally; that is, from their first antenatal care visit, at the glucose challenge test (GCT) time (24-28 weeks of gestation) and at delivery. Passive smoking was self-reported. Macrosomia was defined as birth weight $\geq 4,000 \mathrm{~g}$. Binary logistic regression was used to obtain odds ratios (ORs) and $95 \%$ confidence intervals $(\mathrm{Cls})$. Additive interaction was used to test
\end{abstract}

karger@karger.com www.karger.com/ofa

Karger $\stackrel{\text { ' }}{5}$

GOPEN ACCESS
(C) 2021 The Author(s)

Published by S. Karger AG, Basel

This is an Open Access article licensed under the Creative Commons Attribution-NonCommercial-4.0 International License (CC BY-NC) (http://www.karger.com/Services/OpenAccessLicense), applicable to the online version of the article only. Usage and distribution for commercial purposes requires written permission. the synergistic effect. Results: Passive smokers accounted for $57.4 \%$ of women $(n=8,230)$. Using nonpassive smoking and prepregnancy body mass index (BMI) $<24.0 \mathrm{~kg} / \mathrm{m}^{2}$ as the reference, the adjusted ORs of overweight alone and passive smoking alone for macrosomia were 2.39 (95\% Cl: 2.11-2.71) and 1.17 (95\% Cl: 1.04-1.32). Copresence of passive smoking and prepregnancy $B M I \geq 24.0 \mathrm{~kg} / \mathrm{m}^{2}$ increased the $\mathrm{OR}$ to 2.70 (95\% Cl: 2.28-3.20), with a significant additive interaction. After further adjustment for GDM or GCT, the OR of copresence of both risk factors was slightly attenuated to 2.52 (2.13-3.00) and 2.51 (2.11-2.98), with significant additive interaction. However, the additive interaction between prepregnancy overweight/obesity and passive smoking for LGA was nonsignificant. Conclusions: Prepregnancy overweight/ obesity was associated with an increased risk of macrosomia in Chinese women synergistically with passive smoking during pregnancy, and most of the association was not modified by hyperglycemia during pregnancy.

(C) 2021 The Author(s)

Published by S. Karger AG, Basel

D. Cui and W. Yang equal contribution to the manuscript.
Correspondence to:

Xilin Yang, yxl@ hotmail.com 


\section{Introduction}

Macrosomia is associated with increased risk of adverse pregnancy outcomes such as emergency cesarean section, postpartum hemorrhage, and obstetric anal sphincter injuries for mothers, and shoulder dystocia, obstetrical brachial plexus injury, and fractures for neonates [1]. It also predisposes these infants to a high risk of obesity during their childhood [2].

Fetal macrosomia is one of major concerns of pregnancy complicated by gestational diabetes mellitus (GDM) [3]. In this regard, randomized controlled trials demonstrated that intensive management of women with GDM substantially reduced birth weight and the rate of macrosomia and/or large-for-gestational-age (LGA) infants $[4,5]$. Although management of GDM in China and elsewhere is widespread, a survey showed that the prevalence of macrosomia remained high, for example, $8.5 \%$ in northern China in 2011 [6]. This suggests that many other factors rather than GDM might also contribute to the high prevalence of macrosomia/LGA.

Some studies investigated other risk factors of macrosomia in Western pregnant women and Asian pregnant women $[7,8]$. Maternal overweight/obesity and smoking during pregnancy are 2 well-researched factors for macrosomia. Overweight is consistently found to be associated with fetal overgrowth and macrosomia [7]. On the other hand, the association between maternal smoking and macrosomia is inconclusive. Although a study has shown that exposure to smoking in utero was associated with increased risk of overweight and obesity in children and adolescents, the association of exposure to smoking with early fetal development remains unclear [9]. For example, an early study reported that maternal smoking was associated with decreased risk of macrosomia [8]. It is also noted that maternal smoking was associated with increased risk of low birth weight (LBW) [10]. Both macrosomia and LBW were associated with obesity/overweight later in life [2]. However, the complex interrelationships between maternal overweight/obesity and smoking for the risk of fetal growth and macrosomia are a topic that deserves further investigations.

Although the smoking rate of women was quite low in China, $71.6 \%$ of Chinese women were exposed to secondhand smoking [11]. It has been shown that cigarette smoke increases insulin resistance by altering the distribution of body fat or by exerting a direct toxic influence on pancreatic tissue [12], so that women exposed to passive smoking are more likely to develop GDM. In this context, our group found that passive smoking was asso- ciated with the risk of GDM independently and synergistically with prepregnancy obesity [13]. Given that GDM is an independent risk factor for macrosomia [14] and that prepregnancy obesity and passive smoking can jointly promote the risk of GDM, maternal overweight/obesity and passive smoking may also have an additive interaction on the risk of macrosomia, possibly via increased risk of GDM or hyperglycemia during pregnancy. Therefore, we analyzed the data of the established populationbased cohort of pregnant women in Tianjin, China, to examine (1) the association between exposure to maternal passive smoking and macrosomia; (2) whether there was an additive interaction of prepregnancy overweight/ obesity and passive smoking on risk of macrosomia; and (3) whether the additive interaction was modified by GDM or hyperglycemia during pregnancy.

\section{Materials and Methods}

\section{Study Population and Settings}

The study design, participant characteristics, and data collection process of the study were published previously [15]. Briefly, Tianjin, a gateway to Beijing, is 1 of the 4 cities directly under the administration of the central government of China. It has 6 central urban districts with a population over 4.5 million, where prenatal care was delivered through a 3 -tiered prenatal care system. The prenatal care system consisted of 65 primary care hospitals (the first tier), 6 district-level women and children's health centers and other secondary obstetric hospitals (the second tier), and a citylevel women and children's health center, Tianjin Women and Children's Health Center, and other tertiary obstetric hospitals (the third tier).

\section{Screening for and Diagnosis of GDM}

A two-step GDM screening procedure was utilized to identify GDM cases. All women were offered a 50-g 1-h glucose challenge test (GCT) in nonfasting status from 24 to 28 weeks of gestation, and then a 75-g 2-h oral glucose tolerance test after more than $8 \mathrm{~h}$ of fasting was performed if the GCT result was $\geq 7.8 \mathrm{mmol} / \mathrm{L}$. Fasting plasma glucose, 1-h PG, and 2-h PG were measured immediately using an automatic analyzer (TBA-120FR; Toshiba, Tokyo, Japan) with a coefficient of variance $<2.59 \%$. GDM was diagnosed according to the International Association of Diabetes and Pregnancy Study Group criteria: fasting plasma glucose $\geq 5.1 \mathrm{mmol} / \mathrm{L}$, or $1-\mathrm{h} P G \geq 10.0 \mathrm{mmol} / \mathrm{L}$, or $2-\mathrm{h} \mathrm{PG} \geq 8.5 \mathrm{mmol} / \mathrm{L} \mathrm{[16]}$.

\section{Data Collection and Clinical Measurement}

The data were collected through questionnaires and physical examinations at the time of the first antenatal care visit and at the GCT time (24-28 weeks of gestation) or retrieved from the database of the Maternal and Child Health Information System. At the first antenatal care visit, we collected the information on maternal age, parity, education attainment, and alcohol consumption before/during pregnancy through a questionnaire. Alcohol consumption was queried by an item, "whether you drank alcohol in 
Fig. 1. Flowchart of selection procedure for inclusion of the pregnant women in the analysis.

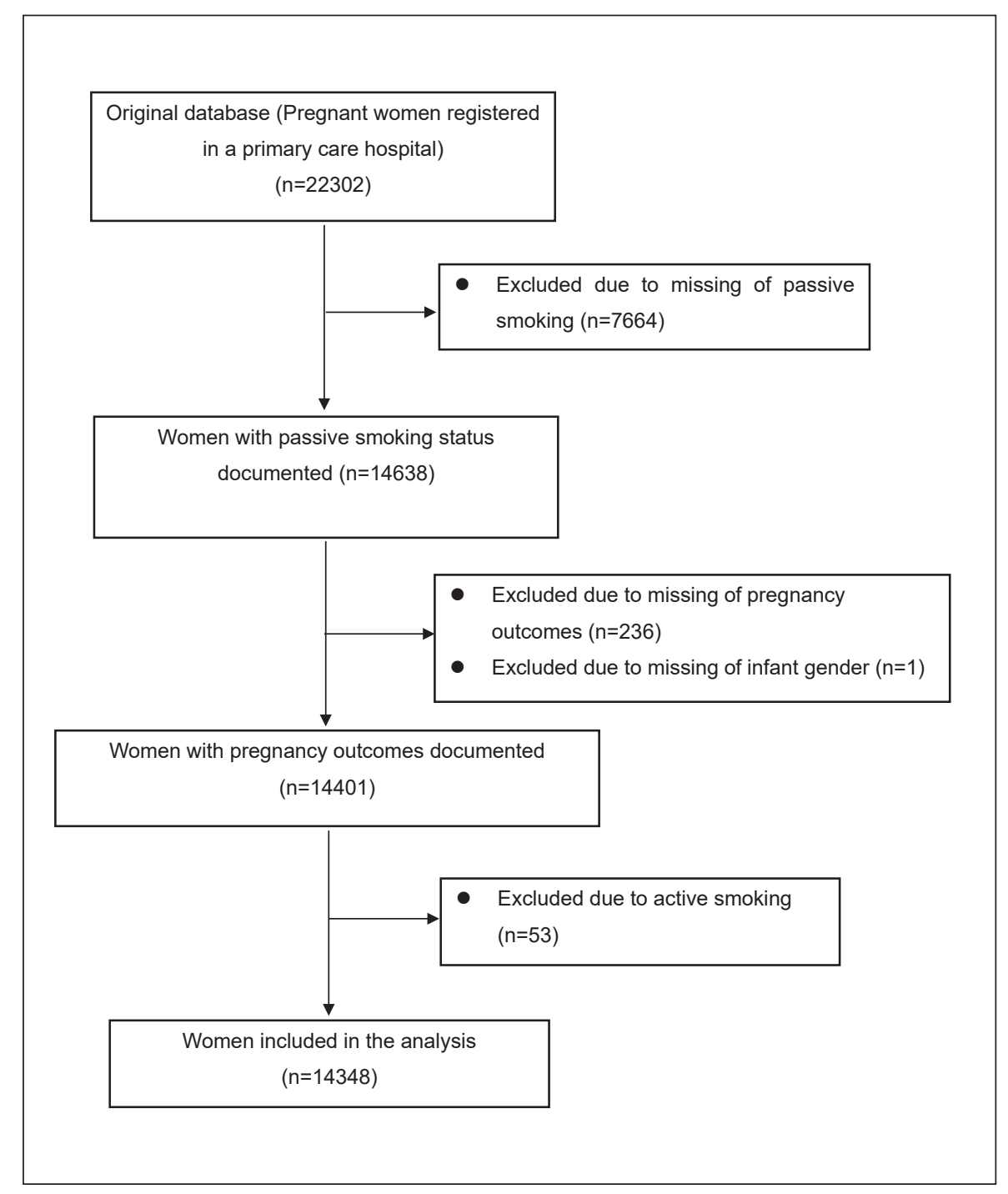

the last 3 months," with 5 response options: "no," "occasionally," "averaging 1-3 days a week," "more than 3 days a week," and "almost every day." An answer "no" was coded as not drinking during pregnancy and any others were coded as alcohol drinker during pregnancy. Education attainment was classified into 2 categories: $>12$ years of schooling and $\leq 12$ years of schooling. After participating in a series of training workshops to standardize the data collection procedures, nurses or clinicians performed anthropometric and clinical measurements at the fieldwork sites. Maternal height and weight were measured without shoes and in light clothing using a beam balance scale (RGZ-120, Jiangsu Suhong Medical Instruments Co., Changzhou, China). Weight at the first antenatal care visit was used as baseline body weight because weight gain during the first 12 weeks of gestation was small [17]. Weight gain from prepregnancy to the GCT time (24-28 weeks of gestation) was calculated as the difference in body weight from the first antenatal care visit to the GCT time, that is, $24-28$ weeks of gestation. Body mass index (BMI) was calculated as weight $(\mathrm{kg})$ divided by the square of body height $(\mathrm{m})$. BMI was categorized into 4 categories: underweight $\left(<18.5 \mathrm{~kg} / \mathrm{m}^{2}\right)$, normal weight $(18.5-23.9 \mathrm{~kg} /$ $\mathrm{m}^{2}$ ), overweight $\left(24.0-27.9 \mathrm{~kg} / \mathrm{m}^{2}\right)$, and obesity $\left(\geq 28.0 \mathrm{~kg} / \mathrm{m}^{2}\right)$ based on the criteria recommended by the Working Group on Obesity in China [18]. Sitting blood pressure was measured after at least 10 min of rest using a calibrated mercury sphygmomanometer at registration. Blood pressure was measured twice at a 5-min interval and the mean value was adopted.

At the time of GCT, that is, 24-28 weeks of gestation, information on passive smoking was obtained through a self-reported questionnaire by asking "Are you exposed to cigarette smoke from others in working and/or living places during your pregnancy?" An answer "Yes" was coded as exposure to passive smoke and "No" was coded as nonexposure to passive smoke. Plasma glucose levels of GCT were measured immediately using an automatic analyzer (TBA-120FR; Toshiba, Tokyo, Japan) with a coefficient of variance $<2.59 \%$. Pregnancy outcome information including birth weight, infant gender, and date of birth were retrieved from an electronical antenatal care management system, the Tianjin Maternal and Child Health Information System, which was established in 2009 and recorded clinical data of all the antenatal care, delivery, and postpartum at the 3 levels of hospitals. 
Table 1. Clinical characteristics of participants by passive smoking status

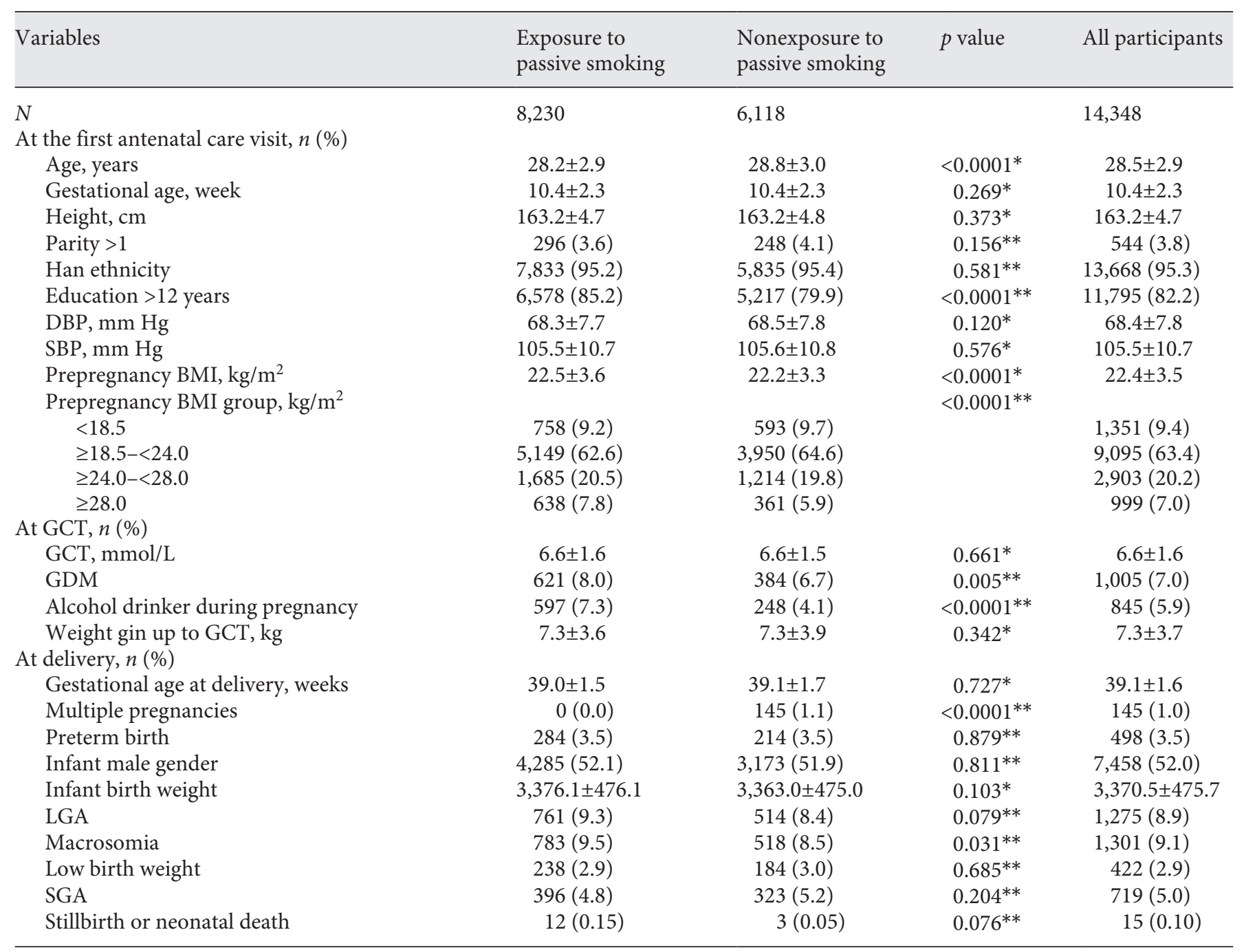

BMI, body mass index; GDM, gestational diabetes mellitus; GCT, glucose challenge test; SBP/DBP, systolic/diastolic blood pressure; LGA, large for gestational age; SGA, small for gestational age. * Derived from Student's $t$ test. ${ }^{* *}$ Derived from $\chi^{2}$ test or Fisher's exact test.

\section{Definition of Clinical Outcomes}

Fetal macrosomia was defined as birth weight being equal to or greater than $4,000 \mathrm{~g}$. LGA was defined as birth weight value greater than the gestational week and gender-specific 90th percentiles according to Tianjin local references.

\section{Statistical Analyses}

Statistical Analysis System, release 9.4 (SAS Institute Inc., Cary, NC, USA) was used to perform all the statistical analyses and a $p$ value $<0.05$ for a 2 -tailed test was considered statistically significant. Continuous variables were presented as mean \pm standard deviation $(\mathrm{SD})$, and categorical variables were presented as number (percentage). When comparing the difference between the passive smoking group and the nonpassive smoking group, Student's $t$ test or Wilcoxon 2-sample test where appropriate was used for con- tinuous variables and $\chi^{2}$ test or Fisher exact test where appropriate was used for categorical variables. Binary logistic regression was used to obtain odds ratios (ORs) and 95\% confidence intervals (CIs) of prepregnancy BMI and passive smoking for macrosomia or LGA (model 1). In multivariable analysis, we adjusted for age, body height, parity, education attainment $>12$ years, gestational age at delivery (only for macrosomia), gender (only for macrosomia), weight gain from prepregnancy to GCT, insulin treatment, alcohol drinker during pregnancy, multiple pregnancies, and systolic blood pressure at first antenatal care visit (model 2). In order to further investigate whether glucose metabolism is an intermediating variable between passive smoking and macrosomia, we further adjusted for the GDM status (model 3) and GCT levels (model 4). We divided the GDM status into 3 groups: (1) the established GDM, (2) GCT $\geq 7.8 \mathrm{mmol} / \mathrm{L}$ but without a subsequent 
Table 2. Odds ratios of prepregnancy overweight/obesity and passive smoking for macrosomia and LGA

\begin{tabular}{|c|c|c|c|c|}
\hline & \multicolumn{2}{|l|}{ Macrosomia } & \multicolumn{2}{|l|}{ LGA } \\
\hline & OR $(95 \% \mathrm{CI})$ & $p$ value & OR $(95 \% \mathrm{CI})$ & $p$ value \\
\hline \multicolumn{5}{|c|}{ Passive smoking versus nonpassive smoking } \\
\hline Model 1 & $1.14(1.01-1.28)$ & 0.0308 & $1.11(0.99-1.24)$ & 0.0593 \\
\hline Model 2 & $1.17(1.04-1.32)$ & 0.0109 & $1.12(1.00-1.27)$ & 0.0569 \\
\hline Model 3 & $1.15(1.02-1.30)$ & 0.0271 & $1.10(0.98-1.25)$ & 0.1225 \\
\hline Model 4 & $1.15(1.02-1.30)$ & 0.0214 & $1.11(0.99-1.25)$ & 0.0980 \\
\hline \multicolumn{5}{|c|}{ Prepregnancy $\mathrm{BMI} \geq 24 \mathrm{~kg} / \mathrm{m}^{2}$ versus $<24 \mathrm{~kg} / \mathrm{m}^{2}$} \\
\hline Model 1 & $2.37(2.11-2.66)$ & $<0.0001$ & $2.39(2.14-2.67)$ & $<0.0001$ \\
\hline Model 2 & $2.39(2.11-2.71)$ & $<0.0001$ & $2.30(2.06-2.58)$ & $<0.0001$ \\
\hline Model 3 & $2.25(1.98-2.55)$ & $<0.0001$ & $2.14(1.89-2.44)$ & $<0.0001$ \\
\hline Model 4 & $2.22(1.96-2.53)$ & $<0.0001$ & $2.13(1.85-2.41)$ & $<0.0001$ \\
\hline \multicolumn{5}{|c|}{$\begin{array}{l}\text { Model 1: univariable analysis. Model 2: multivariable analysis, adjusted for age, height, parity, education }>12 \\
\text { years, gestational age at delivery (only for macrosomia), gender (only for macrosomia), weight gain from pre- } \\
\text { pregnancy to GCT, insulin treatment, alcohol drinker during pregnancy, multiple pregnancies, and systolic blood } \\
\text { pressure at first antenatal care visit. Model 3: further adjusted for GDM, in addition to the variables listed in } \\
\text { model 2. Model 4: further adjusted for GCT value, in addition to the variables listed in model 2. BMI, body mass } \\
\text { index; OR, odds ratio; CI, confidence interval; GDM, gestational diabetes mellitus; GCT, glucose challenge test; } \\
\text { LGA, large for gestational age. }\end{array}$} \\
\hline
\end{tabular}

oral glucose tolerance test, and (3) GCT $<7.8 \mathrm{mmol} / \mathrm{L}$. Subgroup analyses of the association of passive smoking with macrosomia and LGA among participants with and without prepregnancy BMI $\geq 24 \mathrm{~kg} / \mathrm{m}^{2}$ were also performed to test the consistency of ORs of passive smoking for macrosomia and LGA across subgroups.

We used additive interaction to test interactions between maternal prepregnancy $\mathrm{BMI} \geq 24 \mathrm{~kg} / \mathrm{m}^{2}$ and passive smoking during pregnancy for macrosomia or LGA. Three indicators were used to evaluate additive interaction: relative excess risk due to interaction (RERI), attributable proportion due to interaction (AP), or synergy index (S). RERI $>0, A P>0$, or $S>1$ indicated an additive interaction [19]. Separate analyses of the additive interactions between passive smoking and overweight (but not obesity) and between passive smoking and obesity were also performed to check the consistency of the interactions of passive smoking with overweight and obesity for macrosomia.

\section{Results}

\section{Selection of Participants}

Figure 1 is a participant flowchart of the current analysis. From October 2010 to August 2012, 22,302 pregnant women registered for pregnancy at a primary hospital (mean gestational age was $10.4 \pm 2.3$ weeks). Among them, we sequentially excluded 7,664 women with missing passive smoking information, 237 women without information on pregnancy outcomes or infant gender, and 53 active smoking pregnant women. At last, a total of 14,348 pregnant women were included in the final analysis. We had carefully compared the characteristics of the included women and those women excluded but did not find significant differences in maternal age, height, infant gender, BMI, drinking habits, and other features between the 2 groups (online suppl. Table S1; for all online suppl. material, see www.karger.com/doi/10.1159/000517846). Only the parity and the gestational age at the first antenatal visit differed between the 2 groups. The excluded women had a greater proportion of parity $>1$ and older gestational age at the first antenatal visit. The ethics of this study was approved by the Clinical Ethics Committee of Tianjin Women and Children's Health Center and written informed consent was obtained before data collection.

\section{Characteristics of Participants}

Table 1 compared the differences in clinical characteristics of participants by passive smoking status. Among the 14,384 women, $9.1 \%$ and $8.9 \%$ delivered a macrosomic infant or a LGA infant, respectively. At the first antenatal care visit, their mean age was 28.5 (SD: 2.9) years, the mean height was 163.2 (SD: 4.7) cm, and the mean BMI was 22.4 (SD: 3.5$) \mathrm{kg} / \mathrm{m}^{2}$. Of them, 8,230 (57.2\%) women were exposed to passive smoking. The passive smokers had younger maternal age, higher prepregnancy BMI, higher levels of education, and were more likely to have drinking habit and delivered a macrosomic infant or LGA infant. Moreover, women exposed to passive smoking had higher rates of GDM and lower rates of multiple pregnancies. 
Table 3. Interactive associations of passive smoker and prepregnancy overweight/obesity for macrosomia and LGA

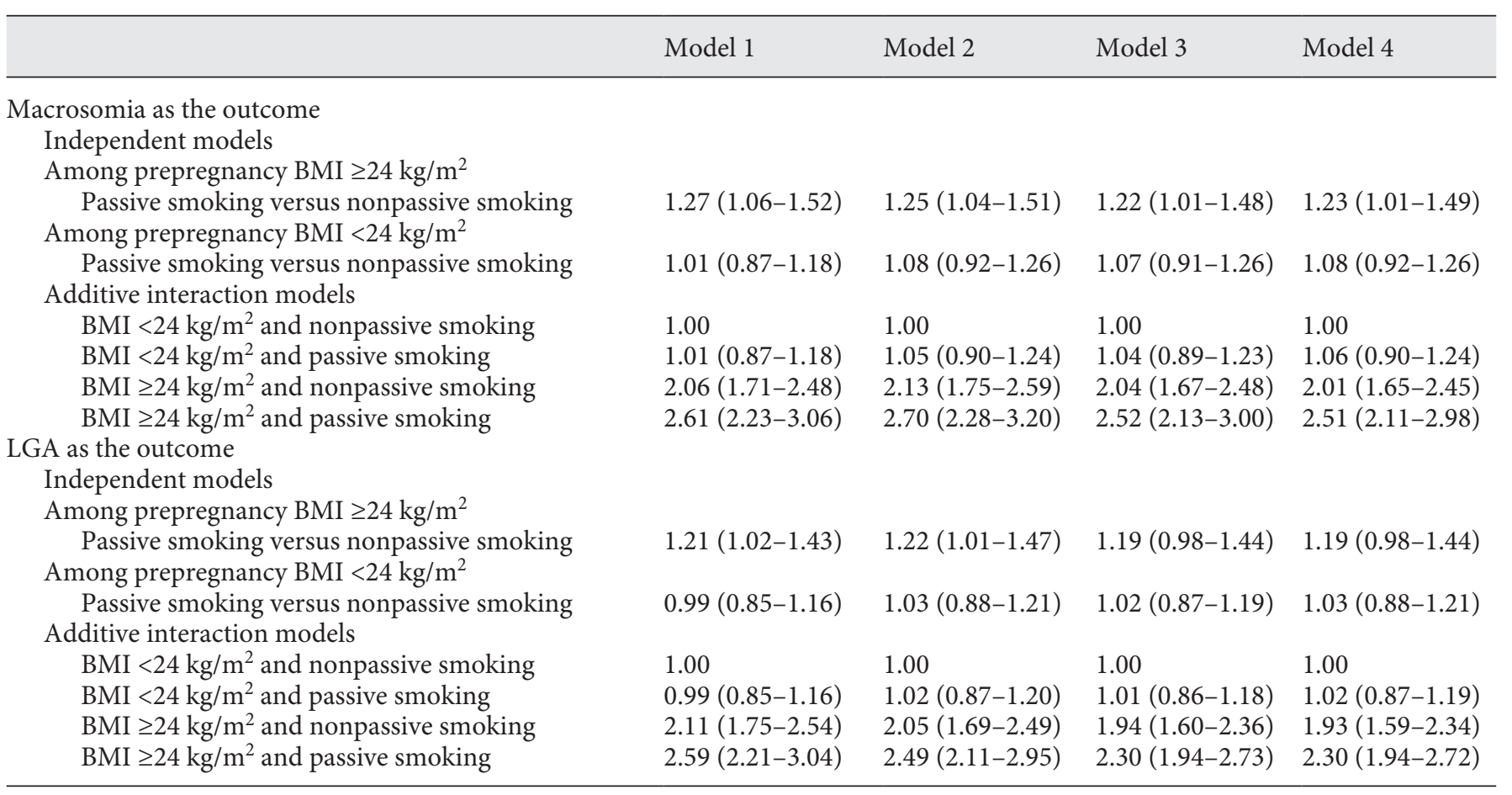

Model 1: univariable analysis. Model 2: multivariable analysis, adjusted for age, height, parity, education $>12$ years, gestational age at delivery (only for macrosomia), gender (only for macrosomia), weight gain from prepregnancy to GCT, insulin treatment, alcohol drinker during pregnancy, multiple pregnancies, and systolic blood pressure at first antenatal care visit. Model 3: further adjusted for GDM, in addition to the variables listed in model 2. Model 4: further adjusted for GCT value, in addition to the variables listed in model 2. BMI, body mass index; LGA, large for gestational age; GDM, gestational diabetes mellitus; GCT, glucose challenge test.

\section{Prepregnancy Overweight and Passive Smoking for Macrosomia or LGA}

Table 2 presents ORs of prepregnancy overweight/ obesity and passive smoking for macrosomia and LGA. The ORs of prepregnancy BMI $\geq 24$ versus $<24 \mathrm{~kg} / \mathrm{m}^{2}$ and passive smoking versus nonpassive smoking for macrosomia were 2.37 (95\% CI: 2.11-2.66) and 1.14 (95\% CI: 1.01-1.28) in univariable analysis, respectively. In the multivariable model (model 2), the ORs of prepregnancy BMI $\geq 24 \mathrm{~kg} / \mathrm{m}^{2}$ and passive smoking for macrosomia were 2.39 (95\% CI: $2.11-2.71)$ and 1.17 (95\% CI: $1.04-$ 1.32), respectively. Adjustment for GDM status (model 3) and GCT levels (model 4) slightly decreased their ORs of passive smoking for macrosomia, but their statistical significance persisted (OR in model 3: 1.15, 95\% CI: 1.02 1.30; OR in model 4: 1.15, 95\% CI: 1.02-1.30). Similarly, adjustment for GDM status and GCT levels also slightly attenuated the association sizes of prepregnancy BMI $\geq 24$ $\mathrm{kg} / \mathrm{m}^{2}$ for macrosomia (OR in model 3: 2.25, 95\% CI: 1.98-2.55; OR in model 4: 2.22, 95\% CI: 1.96-2.53). However, passive smoking was not significantly associated with LGA (except for in model 2), although prepregnancy BMI $\geq 24 \mathrm{~kg} / \mathrm{m}^{2}$ was also associated with increased risk of LGA in all the models.

\section{Additive Interactions between Prepregnancy}

Overweight/Obesity and Passive Smoking for

\section{Macrosomia and LGA}

Table 3 shows the ORs of passive smoking versus nonpassive smoking for macrosomia and LGA among women with BMI $\geq 24 \mathrm{~kg} / \mathrm{m}^{2}$ and $<24 \mathrm{~kg} / \mathrm{m}^{2}$. Among women with prepregnancy BMI $\geq 24 \mathrm{~kg} / \mathrm{m}^{2}$, the OR of passive smoking versus nonpassive smoking for macrosomia was 1.27 (95\% CI: $1.06-1.52)$ in univariable analysis and 1.25 (95\% CI: 1.04-1.51) in multivariable analysis (model 2), both being numerically higher than the ORs in the entire cohort. Similarly, the OR of passive smoking versus nonpassive smoking for LGA among women with prepregnancy BMI $\geq$ versus $<24 \mathrm{~kg} / \mathrm{m}^{2}$ was numerically higher (OR: $1.22,95 \% \mathrm{CI}$ : $1.01-1.47$ ) than among women with BMI $<24.0 \mathrm{~kg} / \mathrm{m}^{2}$ (OR: 1.03, 95\% CI: 0.88-1.21). After adjusting for GDM status (model 3) and GCT glucose levels (model 4), the ORs of 
Table 4. Three measures of the additive interactions between prepregnancy overweight/obesity and passive smoking for macrosomia and LGA

\begin{tabular}{llll}
\hline \multicolumn{1}{c}{ RERI $(95 \% \mathrm{CI})$} & AP $(95 \% \mathrm{CI})$ & $S(95 \% \mathrm{CI})$ \\
\hline $\begin{array}{l}\text { Macrosomia as the outcome } \\
\text { Model 1 }\end{array}$ & $0.54(0.11-0.97)$ & $0.21(0.05-0.36)$ & $1.50(1.03-2.19)$ \\
$\quad$ Model 2 & $0.52(0.05-0.98)$ & $0.20(0.03-0.35)$ & $1.44(1.00-2.06)$ \\
$\quad$ Model 3 & $0.44(0.00-0.89)$ & $0.17(0.01-0.34)$ & $1.40(0.95-2.07)$ \\
Model 4 & $0.44(0.00-0.88)$ & $0.18(0.01-0.34)$ & $1.41(0.96-2.08)$ \\
LGA the outcome & $0.49(0.05-0.93)$ & $0.19(0.03-0.35)$ & $1.44(0.99-2.01)$ \\
$\quad$ Model 1 & $0.42(-0.02$ to 0.85$)$ & $0.16(-0.01$ to 0.33$)$ & $1.39(0.95-2.03)$ \\
Model 2 & $0.35(-0.07$ to 0.76$)$ & $0.15(-0.03$ to 0.33$)$ & $1.36(0.90-2.06)$ \\
Model 3 & $0.35(-0.07$ to 0.76$)$ & $0.15(-0.03$ to 0.33$)$ & $1.36(0.90-2.06)$ \\
Model 4 &
\end{tabular}

Model 1: univariable analysis. Model 2: multivariable analysis, adjusted for age, height, parity, education $>12$ years, gestational age at delivery (only for macrosomia), gender (only for macrosomia), weight gain from prepregnancy to GCT, insulin treatment, alcohol drinker during pregnancy, multiple pregnancies, and systolic blood pressure at first antenatal care visit. Model 3: further adjusted for GDM, in addition to the variables listed in model 2. Model 4: further adjusted for GCT value, in addition to the variables listed in model 2. GDM, gestational diabetes mellitus; CI, confidence interval; LGA, large for gestational age; RERI, relative excess risk due to interaction; $A P$, attributable proportion due to interaction; $S$, synergy index. RERI $>0$, AP $>0$, or $S>1$ suggest significant additive interaction. passive smoking versus nonpassive smoking for macrosomia among women with prepregnancy BMI $\geq 24 \mathrm{~kg} / \mathrm{m}^{2}$ were also numerically higher than among women with prepregnancy BMI $<24 \mathrm{~kg} / \mathrm{m}^{2}$ (Table 3).

Tables 3 and 4 also show the interactive interactions between passive smoker and prepregnancy BMI $\geq 24 \mathrm{~kg} /$ $\mathrm{m}^{2}$ for macrosomia and LGA. Using women with prepregnancy $\mathrm{BMI}<24.0 \mathrm{~kg} / \mathrm{m}^{2}$ and nonpassive smoking as the reference group, the presence of passive smoking increased the ORs of prepregnancy BMI $\geq 24 \mathrm{~kg} / \mathrm{m}^{2}$ for macrosomia from 2.13 (95\% CI: $1.75-2.59)$ to 2.70 (95\% CI: 2.28-3.20) after adjusting for traditional or potential confounding factors including maternal age, height, parity, education $>12$ years, systolic blood pressure, gestational age at delivery, insulin treatment, weight gain from prepregnancy to GCT, multiple pregnancies and alcohol consumption during pregnancy, as well as infant gender. On the other hand, the presence of passive smoking alone was not associated with the increased risk of macrosomia (OR: 1.05, 95\% CI: 0.90-1.24). Table 4 presents the 3 measures of the additive interactions between prepregnancy overweight/obesity and passive smoking for macrosomia and LGA. The measures indicate that the additive interaction for macrosomia was significant (RERI: 0.52, 95\% CI: 0.05-0.98; AP: 0.20, 95\% CI: 0.03-0.35; S: 1.44 , 95\% CI: 1.00-2.06). After further adjustment for GDM status (model 3) or GCT levels (model 4), the interaction between passive smoking and prepregnancy BMI $\geq 24 \mathrm{~kg} / \mathrm{m}^{2}$ persisted and remained significant. On the other hand, the additive interaction between prepregnancy BMI $\geq 24$ $\mathrm{kg} / \mathrm{m}^{2}$ and passive smoking during pregnancy for LGA was nonsignificant (RERI: 0.42, 95\% CI: $-0.02-0.85$; AP: 0.16, 95\% CI: $-0.01-0.33$; S: 1.39, 95\% CI: 0.95-2.03).

\section{Separate Analysis of Additive Interactions between}

Passive Smoking and Overweight (but Not Obesity)

and between Passive Smoking and Obesity for

Macrosomia

Online suppl. Tables S2 and S3 show the results of subgroup analyses of the additive interactions of passive smoking with overweight (but not obesity) and obesity for macrosomia. The OR of copresence of both obesity and passive smoking (OR: 3.56, 95\% CI: 2.78-4.54) was numerically higher than the OR of copresence of overweight and passive smoking (OR: 2.45, 95\% CI: 2.04-2.94). However, only the additive interaction between overweight and passive smoking for macrosomia was significant (RERI: $0.47,95 \% \mathrm{CI}$ : 0.00-0.95; AP: 0.20, 95\% CI: 0.01-0.38; S: $1.51,95 \%$ CI: 0.95-2.40). The additive interaction between obesity and passive smoking did not reach statistical significance.

\section{Additive Interaction between Prepregnancy} Overweight/Obesity and Passive Smoking for Macrosomia after Adjusting for Underweight

Online suppl. Table S4 shows the results of additive interaction between prepregnancy overweight/obesity 
and passive smoking for macrosomia after adjusting for underweight. After adjustment of underweight, the OR of prepregnancy BMI $\geq 24.0 \mathrm{~kg} / \mathrm{m}^{2}$ for macrosomia and the measures for additive interaction between passive smoking and prepregnancy overweight for macrosomia were largely unchanged (RERI: 0.47, 95\% CI: 0.04-0.90; AP: 0.19, 95\% CI: 0.03-0.35; S: 1.46, 95\% CI: 0.99-2.17).

\section{Discussion}

In our study, prepregnancy overweight/obesity and passive smoking were associated with increased risk of macrosomia and exposure to both risk factors had a significant additive interaction toward a higher risk of macrosomia. The interaction between passive smoking and prepregnancy overweight/obesity for macrosomia was not modified by the occurrence of GDM or hyperglycemia during pregnancy. However, passive smoking was not significantly associated with LGA and the additive interaction between both risk factors was also nonsignificant, although prepregnancy overweight/obesity was associated with increased risk of LGA.

There are inconclusive findings regarding the association between maternal smoking during pregnancy and fetal overgrowth and macrosomia. A study found that maternal smoking reduced the risk of macrosomia [8], while others found no crude or adjusted association between maternal smoking and macrosomia [20,21]. Similarly, there were inconsistent findings regarding the association between passive smoking and birth weight [22, 23]. A study conducted in 2 different cities of China found that there were no differences in mean birth weight by exposure from all sources of second-hand smoking [22]. Another study found that the average birth weight among infants whose fathers smoked a pack a day or more was $3,213 \mathrm{~g}$ (95\% CI: 3,025, 3,401) and that among those infants whose fathers did not smoke was 3,191 g (95\% CI: $2,995,3,367)$, a slight increase of $32 \mathrm{~g}$ in average birth weight $(p<0.01)$ [23]. Several studies explored the relationship between passive smoking and small for gestational age (SGA) or LBW, but the results were also inconsistent. Many studies failed to find that second-hand smoke exposure (SHS) was associated with significantly reduced birth weight [24]. However, in a review of 20 studies on the association between second-hand smoke and SGA/LBW, SHS during pregnancy was associated with increased risk of SGA/LBW or significantly reduced birth weight [24]. In the current study, passive smoking exposure was associated with macrosomia. However,

Passive Smoking, Overweight, and Macrosomia passive smoking alone was not associated with increased risk of macrosomia after considering its additive interaction with overweight/obesity for macrosomia.

A large body of literature consistently reported that maternal overweight was associated with increased risk of macrosomia/LGA [25]. A meta-analysis of over 1.6 million Chinese mothers reported that maternal overweight/ obesity before pregnancy was associated with about a 1.91-fold risk of macrosomia [26]. Consistently, we found that prepregnancy overweight/obesity was associated with a 2.39-fold risk of macrosomia and a 2.30 -fold risk of LGA. Our study further found that a large part of the risk association was not attributable to the occurrence of GDM or hyperglycemia during pregnancy, although overweight itself predisposes to a high risk of GDM [27].

There are several mechanisms proposed to explain the association between maternal overweight and fetal overgrowth or macrosomia. Maternal overweight/obesity can change the inflammatory response, resulting in increased concentration of TNF- $a$, IL-1b and IL-6, and leptin and then worsening insulin resistance and fetal overgrowth [28]. Further, a study reported that leptin enhances the activity of the amino acid transporter system A that is among the primary determinants for the supply of nutrients to the fetus [29]. In addition, being overweight/obese also increases the risk of GDM, which further increases the risk of macrosomia. Pedersen's hypothesis states that maternal hyperglycemia can be transported to the fetus via the placenta but insulin could not [30]. The hyperglycemia in the fetus stimulates secretion of insulin in the fetus, which leads to fat accumulation in the fetus [30]. In this context, our group observed that GDM had an interaction with prepregnancy overweight toward increasing the risk of macrosomia [31]. In addition, women without GDM who were overweight before pregnancy also had a higher rate of macrosomia [32].

It is of interest that our current study observed an additive interaction between passive smoking and overweight/obesity for macrosomia independent of GDM or hyperglycemia, suggesting that there are other common mechanisms in addition to glycemia for fetal overgrowth. In this context, a study found that women with GDM whose hyperglycemia was under tight control were still at higher risk of macrosomia [33]. Overweight/obesity is often accompanied by hypertriglyceridemia and insulin resistance [34], a risk factor for macrosomia independent of hyperglycemia [35]. It is more interesting to note that passive smoking increased the odds of maternal overweight/obesity. Our observation is supported by both human and animal studies. Intrauterine exposure to smoking increases the risk of hypertriglyceridemia in adult- 
hood [36]. An animal study found that nondiabetic rats exposed to tobacco smoke had higher triglyceride levels $(515.22 \pm 118.05 \mathrm{mg} / \mathrm{dL})$ than unexposed nondiabetic rats $(352.46 \pm 42.67 \mathrm{mg} / \mathrm{dL})$ [37]. Therefore, our finding regarding the additive interaction between maternal overweight/obesity and passive smoking for macrosomia is biologically plausible.

Our research has public health implications. Macrosomia is associated with increased risk of maternal and neonatal complications including birth injuries, poor 5-min Apgar score, and early neonatal death [38]. Adverse consequences of increased fetal growth may not be limited to the perinatal period because it may increase the risk of diabetes, hypertension, and obesity later in life [39]. In addition, epigenetic changes in embryonic organ development and morphogenesis resulting from high birth weight may contribute to transgenerational programming of chronic disease, and increase the risk of cardiac disease in adults who were born with macrosomia/LGA [40]. Therefore, reducing the incidence of macrosomia has potential public health implications. Overweight/obesity is highly prevalent in Chinese women of reproductive age [41]. The prevalence of overweight/obesity and passive smoking was up to $27.1 \%$ and $57.2 \%$ in our cohort. Given the high rates of passive smoking and maternal overweight, weight control before pregnancy and reducing exposure to passive smoking may reduce the risk of macrosomia. Moreover, passive smoking and overweight/obesity are also associated with increased risk of chronic diseases such as multiple cancers [42, 43], cardiovascular disease, and mortality in later life $[44,45]$. For these reasons, reducing exposure to these 2 factors may have health benefits in both the mothers and their offspring.

Our study had strengths and limitations. A major strength of our study was that our study was a prospective study, and the data were collected longitudinally. Second, our study had excluded all active smokers and adjusted for possible confounders in the analysis. Our study also had limitations. First, a high proportion of women (34.6\%) were not included in the analysis because their passive smoking status was not reported. We had carefully compared the characteristics of the included women and those women excluded but did not find significant differences in most features except for parity and the gestational age at the first antenatal visit (online suppl. Table S1). Second, passive smoking was self-reported rather than objectively measured and self-reported passive smoking may not be an accurate indicator of plasma nicotine levels. However, questionnaires are widely used to assess SHS. Arechavala et al. [46] examined the correla- tion between self-reported SHS exposure indicators and nicotine concentrations in the home and found self-reported SHS exposure indicators correlated moderately strongly with airborne nicotine concentrations (Spearman $r=0.65, p<0.001$ ). Third, we did not systematically collect maternal body weight at birth and we were therefore not able to adjust for weight gain in our analysis. However, we had included weight gain to 24-28 weeks of gestation, that is, at the GCT time, and adjustment of weight gain to 24-28 weeks of gestation may have partially removed the confounding effect of weight gain during pregnancy. Fourth, we used body weight at the first antenatal visit as prepregnancy weight. Although weight gain during the first 12 gestational weeks was quite small [17], use of body weight at the first antenatal care visit for prepregnancy body weight may have resulted in a small overestimation of prepregnancy BMI. Fifth, some information including gestational age at delivery and birth weight was not measured or collected in a prospective manner but retrieved from Tianjin Maternal and Child Health Information System.

In conclusion, our study found that prepregnancy overweight/obesity was associated with increased risk of macrosomia independently and synergistically with passive smoking during pregnancy for macrosomia. A large part of the interactive association was not attributable to hyperglycemia during pregnancy. The underlying mechanism warrants further investigations. Our study highlights the importance of maintaining a healthy weight before pregnancy and reducing exposure to passive smoking for health benefits in the mothers and their offspring.

\section{Acknowledgments}

We express deep thanks to the obstetricians and other health professionals in the 64 primary care hospitals and 6 district women and children's health-care institutes for their participation and contributions to the fieldwork.

\section{Statement of Ethics}

The ethics of this study was approved by the Clinical Ethics Committee of Tianjin Women and Children's Health Center (Approval No. 2009-02), and written informed consent was obtained before data collection.

\section{Conflict of Interest Statement}

All the authors do not have any conflict of interest. 


\section{Funding Sources}

This research was supported by the National Key Research and Development Program of China (Grant No. 2018YFC1313900; 2018YFC1313903) and the National Natural Science Foundation of China (Grant No. 81870549).

\section{Author Contributions}

X.Y. conceived and designed the study. D.C. analyzed the data and D.C. and W.Y wrote the first draft. P.S., P.W., J.L., S.W., and E.L. provided the study material and patients, and collected and assembly the data. J.L., Z.Y., G.H., and J.C. Chan gave critical com- ments on the manuscript. X.Y. (the corresponding author) and D.C. (the first author) take full responsibility for the work as a whole, including the study design, access to data, and the decision to submit and publish the manuscript. All authors have read and approved the submission of the manuscript; the manuscript has not been published and is not being considered for publication elsewhere, in whole or in part, in any language.

\section{Data Availability Statement}

The data are avaiable on reasonable request to the corresponding author.

\section{References}

1 Beta J, Khan N, Khalil A, Fiolna M, Ramadan G, Akolekar R. Maternal and neonatal complications of fetal macrosomia: systematic review and meta-analysis. Ultrasound Obstet Gynecol. 2019 Sep;54(3):308-18.

2 Schellong K, Schulz S, Harder T, Plagemann A. Birth weight and long-term overweight risk: systematic review and a meta-analysis including 643,902 persons from 66 studies and 26 countries globally. PLoS One. 2012; 7(10):e47776

3 Bener A, Saleh NM, Al-Hamaq A. Prevalence of gestational diabetes and associated maternal and neonatal complications in a fast-developing community: global comparisons. Int J Womens Health. 2011;3:367-73.

4 Landon MB, Spong CY, Thom E, Carpenter MW, Ramin SM, Casey B, et al. A multicenter, randomized trial of treatment for mild gestational diabetes. N Engl J Med. 2009 Oct 1; 361(14):1339-48.

5 Crowther CA, Hiller JE, Moss JR, McPhee AJ, Jeffries WS, Robinson JS, et al. Effect of treatment of gestational diabetes mellitus on pregnancy outcomes. N Engl J Med. 2005 Jun 16; 352(24):2477-86.

6 Li G, Kong L, Li Z, Zhang L, Fan L, Zou L, et al. Prevalence of macrosomia and its risk factors in china: a multicentre survey based on birth data involving 101,723 singleton term infants. Paediatr Perinat Epidemiol. 2014 Jul; 28(4):345-50.

7 Liu P, Xu L, Wang Y, Zhang Y, Du Y, Sun Y, et al. Association between perinatal outcomes and maternal pre-pregnancy body mass index. Obes Rev. 2016 Nov;17(11):1091-102.

8 Ju H, Chadha Y, Donovan T, O'Rourke P. Fetal macrosomia and pregnancy outcomes. Aust N Z J Obstet Gynaecol. 2009 Oct;49(5): 504-9.

9 Harris HR, Willett WC, Michels KB. Parental smoking during pregnancy and risk of overweight and obesity in the daughter. Int J Obes. 2013 Oct;37(10): 1356-63.

10 Pereira PP, Da Mata FAF, Figueiredo ACG, de Andrade KRC, Pereira MG. Maternal ac- tive smoking during pregnancy and low birth weight in the Americas: a systematic review and meta-analysis. Nicotine Tob Res. 2017 May 1;19(5):497-505.

11 Xiao L, Yang Y, Li Q, Wang CX, Yang GH. Population-based survey of secondhand smoke exposure in China. Biomed Environ Sci. 2010 Dec;23(6):430-6.

12 Rimm EB, Manson JE, Stampfer MJ, Colditz GA, Willett WC, Rosner B, et al. Cigarette smoking and the risk of diabetes in women. Am J Public Health. 1993 Feb; 83(2):211-4.

13 Leng J, Wang P, Shao P, Zhang C, Li W, Li N, et al. Passive smoking increased risk of gestational diabetes mellitus independently and synergistically with prepregnancy obesity in Tianjin, China. Diabetes Metab Res Rev. 2017 Mar;33(3).

14 He XJ, Qin FY, Hu CL, Zhu M, Tian CQ, Li L. Is gestational diabetes mellitus an independent risk factor for macrosomia: a meta-analysis? Arch Gynecol Obstet. 2015 Apr;291(4): 729-35.

15 Leng J, Shao P, Zhang C, Tian H, Zhang F, Zhang S, et al. Prevalence of gestational diabetes mellitus and its risk factors in Chinese pregnant women: a prospective populationbased study in Tianjin, China. PLoS One. 2015 Mar 23;10(3):e0121029.

16 Alberti KG, Zimmet PZ. Definition, diagnosis and classification of diabetes mellitus and its complications. Part 1: diagnosis and classification of diabetes mellitus provisional report of a WHO consultation. Diabet Med. 1998 Jul; 15(7):539-53.

17 Fattah C, Farah N, Barry SC, O'Connor N, Stuart B, Turner MJ. Maternal weight and body composition in the first trimester of pregnancy. Acta Obstet Gynecol Scand. 2010 Jul;89(7):952-5.

18 Chen C, Lu FC; Department of Disease Control Ministry of Health, PR China. The guidelines for prevention and control of overweight and obesity in Chinese adults. Biomed Environ Sci. 2004;(Suppl 17):1-36.
19 Andersson T, Alfredsson L, Källberg H, Zdravkovic S, Ahlbom A. Calculating measures of biological interaction. Eur J Epidemiol. 2005;20(7):575-9.

20 Strutz KL, Richardson LJ, Hussey JM. Preconception health trajectories and birth weight in a national prospective cohort. J Adolesc Health. 2012 Dec;51(6):629-36.

21 Contreras KR, Kominiarek MA, Zollinger TW. The impact of tobacco smoking on perinatal outcome among patients with gestational diabetes. J Perinatol. 2010 May;30(5):319-23.

22 Lee NL, Samet JM, Yang G, Zhou M, Yang J, Correa A, et al. Prenatal secondhand smoke exposure and infant birth weight in China. Int J Environ Res Public Health. 2012 Sep 26; 9(10):3398-420.

23 Zhang J, Ratcliffe JM. Paternal smoking and birthweight in Shanghai. Am J Public Health. 1993 Feb;83(2):207-10.

24 Hawsawi AM, Bryant LO, Goodfellow LT. Association between exposure to secondhand smoke during pregnancy and low birthweight: a narrative review. Respir Care. 2015 Jan;60(1):135-40.

25 Yu Z, Han S, Zhu J, Sun X, Ji C, Guo X. Prepregnancy body mass index in relation to infant birth weight and offspring overweight/ obesity: a systematic review and meta-analysis. PLoS One. 2013 Apr 16;8(4):e61627.

26 Liu L, Ma Y, Wang N, Lin W, Liu Y, Wen D. Maternal body mass index and risk of neonatal adverse outcomes in China: a systematic review and meta-analysis. BMC Pregnancy Childbirth. 2019 Mar 29;19(1):105.

27 Najafi F, Hasani J, Izadi N, Hashemi-Nazari SS, Namvar Z, Mohammadi S, et al. The effect of prepregnancy body mass index on the risk of gestational diabetes mellitus: a systematic review and dose-response meta-analysis. Obes Rev. 2019 Mar;20(3):472-86.

28 Westermeier F, Sáez PJ, Villalobos-Labra R, Sobrevia L, Farías-Jofré M. Programming of fetal insulin resistance in pregnancies with maternal obesity by ER stress and inflammation. Biomed Res Int. 2014;2014:917672. 
29 Jansson N, Greenwood SL, Johansson BR, Powell TL, Jansson T. Leptin stimulates the activity of the system A amino acid transporter in human placental villous fragments. J Clin Endocrinol Metab. 2003 Mar; 88(3): 1205-11.

30 Pedersen J. Weight and length at birth of infants of diabetic mothers. Acta Endocrinol. 1954 Aug; 16(4):330-42.

31 Yang W, Liu J, Li J, Liu J, Liu H, Wang Y, et al. Interactive effects of prepregnancy overweight and gestational diabetes on macrosomia and large for gestational age: a population-based prospective cohort in Tianjin, China. Diabetes Res Clin Pract. 2019 Aug;154: 82-9.

32 Ijäs H, Koivunen S, Raudaskoski T, Kajantie E, Gissler M, Vääräsmäki M. Independent and concomitant associations of gestational diabetes and maternal obesity to perinatal outcome: a register-based study. PLoS One. 2019 Aug 29;14(8):e0221549.

33 Olmos PR, Borzone GR, Olmos RI, Valencia $\mathrm{CN}$, Bravo FA, Hodgson MI, et al. Gestational diabetes and pre-pregnancy overweight: possible factors involved in newborn macrosomia. J Obstet Gynaecol Res. 2012 Jan;38(1): 208-14.

34 Barrett HL, Dekker Nitert M, McIntyre HD, Callaway LK. Normalizing metabolism in diabetic pregnancy: is it time to target lipids? Diabetes Care. 2014 May;37(5):1484-93.
35 Gorban de Lapertosa S, Alvariñas J, Elgart JF, Salzberg S, Gagliardino JJ; EduGest group. The triad macrosomia, obesity, and hypertriglyceridemia in gestational diabetes. Diabetes Metab Res Rev. 2020 Jul;36(5):e3302.

36 Cupul-Uicab LA, Skjaerven R, Haug K, Travlos GS, Wilson RE, Eggesbø M, et al. Exposure to tobacco smoke in utero and subsequent plasma lipids, ApoB, and CRP among adult women in the MoBa cohort. Environ Health Perspect. 2012 Nov; 120(11):1532-7.

37 de Souza Mda S, Sinzato YK, Lima PH, Calderon IM, Rudge MV, Damasceno DC. Oxidative stress status and lipid profiles of diabetic pregnant rats exposed to cigarette smoke. Reprod Biomed Online. 2010 Apr;20(4):547-52.

38 Nkwabong E. Maternal and neonatal complications of macrosomia. Trop Doct. 2014; 44(4):201-4.

39 Tian JY, Cheng Q, Song XM, Li G, Jiang GX, $\mathrm{Gu}$ YY, et al. Birth weight and risk of type 2 diabetes, abdominal obesity and hypertension among Chinese adults. Eur J Endocrinol. 2006 Oct;155(4):601-7.

40 Lin XH, Wu DD, Gao L, Zhang JY, Pan HT, Wang $\mathrm{H}$, et al. Altered DNA methylation in neonates born large-for-gestational-age is associated with cardiometabolic risk in children. Oncotarget. 2016 Dec 27;7(52):8651121.
41 Li N, Liu E, Guo J, Pan L, Li B, Wang P, et al. Maternal prepregnancy body mass index and gestational weight gain on pregnancy outcomes. PLoS One. 2013 Dec 20;8(12):e82310.

42 Taylor R, Najafi F, Dobson A. Meta-analysis of studies of passive smoking and lung cancer: effects of study type and continent. Int J Epidemiol. 2007 Oct;36(5):1048-59.

43 Neuhouser ML, Aragaki AK, Prentice RL, Manson JE, Chlebowski R, Carty CL, et al. Overweight, obesity, and postmenopausal invasive breast cancer risk: a secondary analysis of the women's health initiative randomized clinical trials. JAMA Oncol. 2015 Aug;1(5): 611-21.

44 Lee W, Hwang SH, Choi H, Kim H. The association between smoking or passive smoking and cardiovascular diseases using a Bayesian hierarchical model: based on the 2008 2013 Korea community health survey. Epidemiol Health. 2017 Jun 22;39:e2017026.

45 Lavie CJ, Milani RV, Ventura HO. Obesity and cardiovascular disease: risk factor, paradox, and impact of weight loss. J Am Coll Cardiol. 2009 May 26;53(21):1925-32.

46 Arechavala T, Continente X, Pérez-Ríos M, Fernández E, Cortés-Francisco N, Schiaffino A, et al. Validity of self-reported indicators to assess secondhand smoke exposure in the home. Environ Res. 2018 Jul;164:340-5. 